\title{
MINIREVIEW
}

\section{The THO complex as a key mRNP biogenesis factor in development and cell differentiation}

\author{
Sonia Jimeno and Andrés Aguilera* \\ See research article http://www.biomedcentral.com/1741-7007/8/1
}

\begin{abstract}
The THO complex is a key component in the co-transcriptional formation of messenger ribonucleoparticles that are competent to be exported from the nucleus, yet its precise function is unknown. A recent study in BMC Biology on the role of the THOC5 subunit in cell physiology and mouse development provides new clues to the role of the THO complex in cell differentiation.
\end{abstract}

As soon as the pre-mRNA has been transcribed from DNA in the nucleus, it is processed into a mature ribonucleoprotein (mRNP) particle, which is competent to be exported from the nucleus. The THO complex, a nuclear protein complex conserved from yeast to humans, is involved in the biogenesis of mRNP particles and functions at the interface between transcription and RNA export (Figure 1).

Although it is now clear that the THO complex has a role in RNA metabolism, the initial studies that ended in the identification of this key complex had nothing to do with transcription, mRNP biogenesis or RNA export. The first known component of THO, Hpr1, was identified through a genetic screen for hyper-recombinant mutants in Saccharomyces cerevisiae. Subsequent genetic and molecular characterization of mutants in which the hpr1

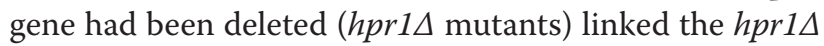
hyper-recombination phenotype to transcription and showed that Hpr1 was involved in transcriptional elongation. Tho2 was then identified as a high-copynumber suppressor of hpr $1 \Delta$. Yeast tho $2 \Delta$ mutants also showed a strong hyper-recombination phenotype that was linked to a transcription elongation defect (reviewed in [1]).

\footnotetext{
*Correspondence: aguilo@us.es

Centro Andaluz de Biología Molecular y Medicina Regenerativa, Av. Américo Vespucio s/n, 41092 Sevilla, Spain
}

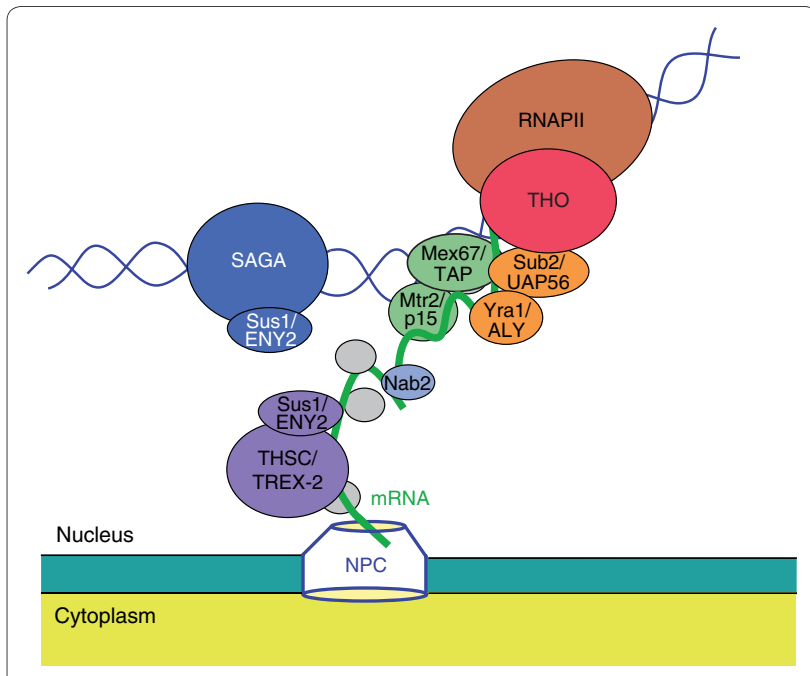

Figure 1. The THO complex functions in mRNP biogenesis at the interface between transcription and export of mRNA from the nucleus. Proteins are shown with their yeast name followed by the name of the human homolog, where the two differ, for Nab2, Mtr2/p15, Sub2/UAP56, Mex67/TAP, Yra1/ALY, or with the yeast name followed by the Drosophila name for Sus1/ENY2. Protein complexes are shown in capital letters: THO, THSC (also called TREX-2) and SAGA. Proteins that interact with each other or between which a physical connection has been reported are in the same color. Sus 1 can act as a subunit of both THSC and SAGA complexes. Unlabeled proteins in gray represent other factors important for mRNP biogenesis and export. NPC, nuclear pore complex; RNAPII, RNA polymerase II.

Hyper-recombination could have been seen as just a side effect of the physiological consequences of THO mutations, but it turned out to reveal a role for THO in forming an optimal mRNP particle, one that prevents the nascent RNA from interacting with the DNA template. In hpr1S mutants, the nascent RNA forms an RNA-DNA hybrid (R-loop) with the DNA template strand, while the other DNA strand remains single stranded; the formation of such 'R-loops' is linked to hyper-recombination (reviewed in [1]). Research from an increasing number of laboratories has revealed THO to be a conserved nuclear factor with a key function in mRNP biogenesis and export as well as in development and cell differentiation. A recently reported analysis [2] of a conditional knockout 
mouse of the THOC5 subunit of THO adds new perspectives to the role of $\mathrm{THO}$ in differentiation.

\section{THO as a conserved physical and functional unit with a role in $\mathrm{mRNP}$ biogenesis}

The yeast THO complex was first purified from Saccharomyces cerevisiae with a tagged His(6)-Tho2 under high-salt conditions as a robust four-subunit complex formed by Tho2, Hpr1, Mft1 and Thp2. Null mutations in all THO components confer the same phenotypes - transcription impairment, hyperrecombination and defective RNA export - indicating that THO is a functional and physical unit. Further purification of the THO complex together with the mRNA export factors Yra1 and Sub2, the latter of which is an RNA-dependent ATPase involved in mRNA export, in a larger complex termed TREX (transcription-export complex), and the identification of Sub2 as a high-copy suppressor of $h p r 1 \Delta$ led to the connection of THO with RNA export (reviewed in [1]). This conclusion was strengthened by the observation that sub2 mutants led to a similar transcription-dependent hyper-recombination phenotype to that of THO-complex mutants and that these also show RNA-export defects. Nevertheless, the physical interactions among the THO components are much stronger than those with other components of TREX. THO is stable in high salt conditions in the absence of Yra1 and in which Sub2 is present in trace amounts that can be detected only by western blotting (reviewed in [1]). Indeed, the integrity of the yeast THO complex requires Hpr1, Tho2, Mft1 and Thp2 but not Sub2 [3]. The human or Drosophila THO complexes also contain hTho2/THOC2, hHpr1/THOC1, Tex1/THOC3 and three additional subunits called THOC5, THOC6 and THOC 7. The Sub2 ortholog UAP56 can be detected in low amounts in Drosophila and is absent in human cells immunodepleted of hTho2, indicating that in these organisms the core THO complex exists as a salt-resistant stable complex independent of UAP56 and Yra1 [4,5].

In yeast, THO binds to active chromatin in an RNAindependent manner. A plausible scenario is as follows (Figure 1): THO could be one of the first players to act during transcription elongation to facilitate a correct mRNP formation helping recruit other factors, such as Sub2 or Mex67 [6]. Other RNA binding proteins, such as Yra1, which interacts with Sub2, and the Mex67-Mtr2 export factor, could act at subsequent steps in this scenario to bring the mRNP to the nuclear pore complex. THO helps recruit Mex67 to the mRNP through Hpr1, an interaction that is regulated by Rsp5, an ubiquitin ligase that polyubiquitinates $\mathrm{Hpr} 1$ [7]. Close to the nuclear pore complex, the THSC complex, also termed TREX-2, could have an as-yet unknown function in mRNP biogenesis and export. Interestingly, mutations in
THSC confer the same phenotypes of transcription elongation impairment, defective RNA export and transcription-dependent hyper-recombination as do THO mutations (reviewed in [1]).

Human THO associates with proteins of the spliceosome and with spliced RNAs, this latter interaction being independent of transcription $[4,8]$. However, there is also evidence for transcription-dependent recruitment of THO to chromatin in Drosophila [9]. The role of THO in mRNP metabolism therefore may be general among eukaryotes. The recent observation that Drosophila THO complex interacts with ENY2, a protein previously identified as a transcriptional activator that interacts with the SAGA transcription factor, opens up the possibility of a co-transcriptional action of THO in higher eukaryotes [9]. The impact of THO in RNA physiology, however, may go beyond transcription elongation and its associated RNA metabolism steps, as shown by the involvement of THO in mRNA 3' end processing, whether or not direct, and by the identification in yeast THO mutants of a larger nuclear macromolecular structure containing components of the nuclear pore complex and polyadenylation factors [10].

\section{Function of THO in development and differentiation}

The relevance of THO in cell physiology has been clearly shown from yeast to humans. Yeast THO null mutants are sick and slow growers and THO depletion has a negative effect on growth rate of human and Drosophila cell lines. THO is required for viability of the early mouse embryo and for postnatal survival, as determined by a THOC1 knockout $[2,11]$. Whether the relevance of THO function is a consequence of a general, genome-wide role or whether its role is limited to a subset of genes is still an open question. In humans and Drosophila, various studies have shown that $\mathrm{THO}$ is required for the export of heat shock mRNAs, but nothing is known about other mRNAs. Similarly, it is unknown whether THO function is required equally in different cell tissues and throughout development and differentiation. Importantly, however, THOC1 conditional knockout mice reveal abnormal testis development that causes sterility [12]. Understanding the relevance of THO in development and tissue differentiation as well as its putative impact in cancer and cell proliferation should provide a better understanding of its functional role.

One step towards this understanding is the report by Mancini et al. [2], who have constructed and characterized an interferon-inducible cre-recombinase based conditional THOC5 knockout mouse. THOC5 deletion causes death in the first 2 weeks, similar to THOC1 deletion. Mice with the conditional knockout develop acute leukocytopenia (a reduction in white blood cell 
numbers) and anemia (a reduction in red blood cell numbers). The number of blood cells in peripheral blood is reduced drastically; this is caused by apoptosis of bone marrow cells and loss of committed myeloid progenitor cells and of cells with long-term reconstituting potential. The transfer of normal bone marrow cells rescued $70 \%$ of the mice from death. The data [2] support the hypothesis that THOC5 is critical in bone marrow and in hematopoiesis, but not for hepatocytes and heart muscles. The results are consistent with previous work from this same group (referenced in [2]) showing that human THOC5 affects granulocyte/macrophage differentiation and adipocyte differentiation, and further support the idea that the THO complex has a key role not only in early embryogenesis, but also in differentiation, as previously reported with THOC1 knockout mice $[11,12]$.

In the near future it would be interesting to know whether the subunits of the THO complex have different roles in the differentiation of distinct cell types. And it would certainly be important to understand how the role of the THO complex in development and differentiation is related to its molecular function in mRNP biogenesis and export. Among the various possibilities that would need to be investigated to understand the role of this intriguing complex in differentiation are whether or not the THO complex has specific functions in different cell types and how this might be related to particular functions that THO could have for specific target mRNAs or depending on the putative THO protein modifications (phosphorylation, ubiquitination, and so on) that might change its pattern of activity.

\section{Acknowledgements}

We thank R Luna and AG Rondón for critical reading of the manuscript. The work of AA's laboratory is funded by the Spanish Ministry of Innovation and Junta de Andalucía.

Published: 28 January 2010
References

1. Aguilera A: Cotranscriptional mRNP assembly: from the DNA to the nuclear pore. Curr Opin Cell Biol 2005, 17:242-250.

2. Mancini A, Niemann-Seyde SC, Pankow R, El Bounkari O, Klebba-Farber S, Koch A, Jaworska E, Spooncer E, Gruber AD, Whetton AD, Tamura T: THOC5/FMIP, an mRNA export TREX complex protein, is essential for hematopoietic primitive cell survival in vivo. BMC Biol 2010, 8:1.

3. Huertas P, García-Rubio ML, Wellinger RE, Luna R and Aguilera A: An hpr 1 point mutation that impairs transcription and mRNP biogenesis without increasing recombination. Mol Cell Biol 2006, 26:7451-7465.

4. Masuda S, Das R, Cheng H, Hurt E, Dorman N, Reed R: Recruitment of the human TREX complex to mRNA during splicing. Genes Dev 2005, 19:1512-1517.

5. Rehwinkel J, Herold A, Gari K, Kocher T, Rode M, Ciccarelli FL, Wilm M, Izaurralde E: Genome-wide analysis of mRNAs regulated by the THO complex in Drosophila melanogaster. Nat Struct Mol Biol 2004, 11:558-566.

6. Zenklusen D, Vinciguerra P, Wyss JC, Stutz F: Stable mRNP formation and export require cotranscriptional recruitment of the mRNA export factors Yra1p and Sub2p by Hpr1p. Mol Cell Biol 2002, 22:8241-8253.

7. Hobeika M, Brockmann C, Gruessing F, Neuhaus D, Divita G, Stewart M, Dargemont C: Structural requirements for the ubiquitin-associated domain of the mRNA export factor Mex67 to bind its specific targets, the transcription elongation THO complex component Hpr1 and nucleoporin FXFG repeats. J Biol Chem 2009, 284:17575-17583.

8. Cheng H, Dufu K, Lee CS, Hsu JL, Dias A and Reed R: Human mRNA export machinery recruited to the $5^{\prime}$ end of mRNA. Cell 2006, 127:1389-1400.

9. Kopytova DV, Orlova AV, Krasnov AN, Gurskiy DY, Nikolenko JV, Nabirochkina EN, Shidlovskii YV, Georgieva SG: Multifunctional factor ENY2 is associated with the THO complex and promotes its recruitment onto nascent mRNA. Genes Dev 2010, 24:86-96.

10. Rougemaille M, Dieppois G, Kisseleva-Romanova E, Gudipati RK, Lemoine S, Blugeon C, Boulay J, Jensen TH, Stutz F, Devaux F, Libri D: THO/Sub2p functions to coordinate 3 '-end processing with gene-nuclear pore association. Cell 2008, 135:308-321.

11. Wang $X$, Chang $Y$, Li Y, Zhang $X$, Goodrich DW: Thoc1/Hpr1/p84 is essential for early embryonic development in the mouse. Mol Cell Biol 2006, 26:4362-4367.

12. Wang X, Chinnam M, Wang J, Wang Y, Zhang X, Marcon E, Moens P, Goodrich DW: Thoc1 deficiency compromises gene expression necessary for normal testis development in the mouse. Mol Cell Biol 2009, 29:2794-2803.

doi:10.1186/jbiol217

Cite this article as: Jimeno S, Aguilera A: The THO complex as a key mRNP biogenesis factor in development and cell differentiation. Journal of Biology 2010, 9:6. 